1

19 *Corresponding Author:-

20 Prof. (Mrs.) Chandra Mohini Chaturvedi,

21 Department of Zoology, Banaras Hindu University

22 Varanasi-221 005, U.P., INDIA,

23 Phone No. 0542-6702506, 9415819743

24 Fax: 0542-6702506

25 E-mail: suneeta17bhu@gmail.com, cmcbhu@gmail.com

\section{Japanese quail}

26 


\section{Abstract}

30 Specific temporal phase relation of serotonergic and dopaminergic oscillations alters

31 reproductive responses in many species. Aim of the study was to confirm whether effect 32 of serotonergic drug (5-HTP) and dopaminergic drug (L-DOPA) is due to their 33 conversion into serotonin and dopamine respectively or other products. For this study, 34 PCPA (p-chlorophenylalanine, a long lasting inhibitor of serotonin synthesis), DDC 35 (Diethyldithiocarbamate, which inhibits biosynthesis of nor-adrenaline), $\alpha$-MT (Methyl36 p-tyrosine, an inhibitor for the conversion of tyrosine to DOPA) and DOPS 37 (Dihydroxyphenylserine, a specific precursor for noradrenaline) were used in different 38 groups in addition to 5-HTP and L-DOPA given at specific time interval. Reproductive 39 responses monitored at 10 weeks post treatment indicate that gonadal activity was 40 significantly low in HTP:DOPA (8-hr quail), HTP+PCPA:DOPA and 41 HTP:DOPA+DDC quail compare to control (S:S). However, gonadal activity of 42 HTP:S(HTP control), S:DOPA(DOPA control) and HTP: $\alpha-M T+D O P S$ was not 43 different from $\mathrm{S}: \mathrm{S}$ control and remained in active condition. These findings indicate 44 that it is not the dose of neurotransmitter precursor drugs (5-HTP and L-DOPA) and the 45 neurotransmitters (serotonin and dopamine itself) that cause the effect, instead it is the 46 function of interval between the drug administration which induces or entrains specific 47 phase relation between serotonergic and dopaminergic oscillations. Further, gonadal 48 suppression observed in HTP:DOPA, HTP+PCPA:DOPA and HTP:DOPA+DDC group 49 three groups is not due to injection of 5-HTP or L-DOPA (alone) but due to conversion 50 of administered 5-HTP into serotonin and conversion of L-DOPA (administered) into 51 dopamine; not due to their further conversion into catecholamines other than dopamine 52 i.e. noradrenaline or adrenaline.

Keywords: 5-HTP; L-DOPA; Specific temporal phase relation; Serotonergic and 
Introduction

71 Owasoyo and Walker, 1980) shows an opposite circadian pattern with peak levels

72 occurring during light phase of the light:dark cycle. It has also been shown that brain

73 serotonin and other neurotransmitters (dopamine) exhibit circadian variation in different

74 brain areas including suprachiasmatic nuclei (SCN) (Philo et al., 1977; Héry et al.,

75 1981; Wilson and Meier, 1987; 1988). Experimentally it has been demonstrated that the

76 circadian rhythms exist in hormones as well as neurotransmitters viz. 5-HT, NE and DA

77 (Manshardt and Wurtman, 1968; Wilson and Meier, 1987; Forsling, 2000; Tiwari et al.,

78 2006). A number of studies also indicate the circadian release of 5-HT in blood and

79 pineal of mammals, birds and fish (Reis and Wurtman, 1968; Reis et al., 1969). Thus,

80 there appears to be considerable synchrony throughout the brain with regards to the

81 rhythms of neurotransmitters content and activity (Reis et al., 1969; Le Bras, 1984;

82 Khan and Joy, 1990).

83 It has been well established from several reports that administration of 5-HTP and L-

84 DOPA at the interval of $12 \mathrm{hrs}$ stimulates gonadal growth and body weight gain

85 whereas administration of these drugs at the interval of $8 \mathrm{hrs}$ results into opposite effect

86 i.e. gonadal suppression in several avian (Red headed bunting- Chaturvedi and Bhatt,

87 1990; Bhatt and Chaturvedi, 1992b; Phillips and Chaturvedi, 1992; Lal munia-

88 Chaturvedi et al., 1994; Japanese quail- Chaturvedi et al., 1991; Bhatt and Chaturvedi,

89 1992a; Phillips and Chaturvedi, 1995; Bhatt and Chaturvedi, 1998; Tiwari and 
90 Chaturvedi, 2003; Chaturvedi et al., 2006; Kumar and Chaturvedi, 2008; Indian weaver

91 bird- Chaturvedi et al., 1997; Spotted munia- Chaturvedi and Prasad, 1991; Prasad and

92 Chaturvedi, 1992a, 1992b, 1992c, 2003) and mammalian species whether seasonally

93 breeding (Syrian hamster- Wilson and Meier, 1989; Indian palm squirrel- Chaturvedi

94 and Jaiwal, 1990; Jaiwal and Chaturvedi, 1991; Chaturvedi and Singh, 1992) or

95 continuous breeder (laboratory mouse, Mus musculus- Sethi and Chaturvedi, 2009;

96 Sethi et al, 2010).

In some of these studies, instead of 5-HTP and L-DOPA given at specific time interval, each drug was given in combination with saline (5-HTP \& saline or saline \& L-DOPA) (Prasad and Chaturvedi, 2003). Since these combinations did not produce any significant effect or long lasting effect, it was suggested that it is not the effect of 101 either serotonin or dopamine alone, but actually the interval between the 102 administrations of two drugs is important to mimic the seasonal gonadal condition. It was presumed and later on also proved experimentally that, these timed injection of serotonin and dopamine precursor drugs given for a period of 11-13 days may entrain circadian serotonergic and dopaminergic oscillation respectively. Moreover, the two precursor drugs given at different time interval will induce different phase relationship or phase angle between the two oscillations and different physiology (Yadav and

108 Chaturvedi, 2014, 2015). Obviously, in nature also during sexually active and inactive 109 condition these phase relations vary the underlying basis of our hypothesis and all the experimental studies in this direction (Wilson and Meier, 1987, 1988, 1989; Tiwari and

111 Chaturvedi, 2003; Tiwari et al., 2006). In view of fact that neurotransmitters serotonins 112 and dopamine do not cross the blood brain barrier but their precursor do cross 113 (Bianchine, 1980) in all these studies 5-HTP and L-DOPA were used as the precursor 114 of serotonin and dopamine respectively instead of neurotransmitter itself. 

effects are due to temporal phase relationship of circadian neural oscillations and not

117 due to serotonin and dopamine alone (Prasad and Chaturvedi, 2003). L-DOPA not only

118 gets converted into dopamine in the presence of enzyme tyrosine hydroxylase but the 119 next biosynthetic product is the noradrenaline (by enzyme dopamine hydroxylase) and 120 adrenaline (by enzyme N-methyl transferase) respectively. Further, tryptophan 121 converts into 5-HTP (by tryptophan hydroxylase) and 5-HTP gets converted into 122 serotonin (by enzyme aromatic amino acid decarboxylase) and next product is 123 melatonin in certain tissues. This experiment was conducted to confirm that effects 124 observed in the earlier studied are due to conversion of 5-HTP into serotonin and that of 125 L-DOPA into dopamine only and not their next biosynthetic products. Hence agonist 126 and antagonists of different enzymes were used an addition to 5-HTP and L-DOPA 127 given at specific time interval to study the reproductive response of Japanese quail. The 128 biosynthetic pathway of neurotransmitter serotonin and dopamine, conversion of 129 dopamine into other catecholamines and agonists and antagonists of these biosynthetic 130 products are described below in details along with the line diagram.

131 1. PCPA (p-chlorophenylalanine), a long lasting inhibitor of serotonin synthesis (Koe 132 and Weiseman, 1966). It blocks the conversion of tryptophan into 5-HTP by 133 inactivating the enzyme tryptophan hydroxylase. It does not affects the conversion of 5134 HTP into serotonin.

135 2. DDC (Diethyldithiocarbamate), which inhibits the conversion of Dopamine to nor136 adrenaline by inducing negative effect on the enzyme Dopamine B-hydroxylase, 137 essential for this biosynthesis (Crevelling et al., 1968). 
138 3. $\alpha$-MT (Methyl-p-tyrosine). An inhibitor for the conversion of tyrosine to DOPA by

139 affecting the enzyme tyrosine hydroxylase. enhances the synthesis of noradrenaline.
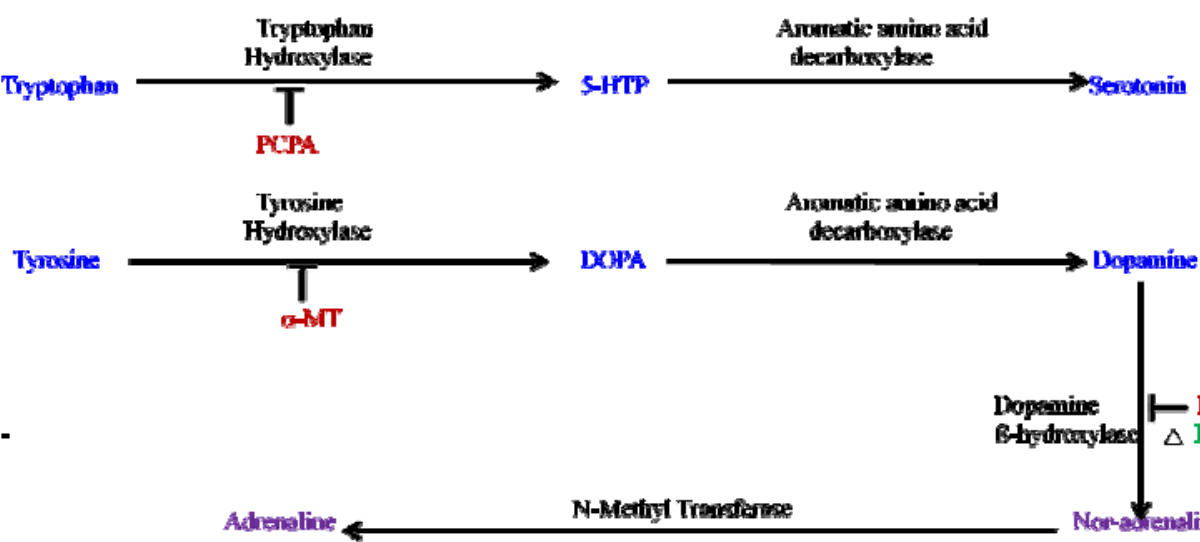

The specific aim of the present study was to prove that stimulation (due to $12 \mathrm{hr}$

144 phase relation) or regression (due to $8 \mathrm{hr}$ phase relation) of gonadal development is due

145 to specific temporal phase relation of serotonergic and dopaminergic activities or 146 oscillations and not due to i) serotonin/5-HTP or dopamine/L-DOPA alone and ii) that

147 L-DOPA was effective when converted into dopamine and not into noradrenaline or

148 adrenaline. For the above mentioned objectives, some agonist/antagonists of serotonin 149 (PCPA) and Dopamine ( $\alpha$-MT, DDC, DOPS) were used in combination with these 150 neurotransmitters (serotonin and dopamine) precursors (5-HTP and L-DOPA).

\section{Materials and methods}

152 Three week old male Japanese quail purchased from Chuck Gazaria farm, 153 Lucknow, were divided into 7 groups each having 8 birds. 
155 I (Saline control/S:S)-

156

157 II (HTP control/5-HTP:S)-

158

159

160

161

162

163

164

165

166

167

168

169

170

171

172

173

174

175

176

177

178

179

IV (HTP:DOPA)-

V (HTP+PCPA:DOPA) -

VI (HTP:DOPA+DDC)-

VII (HTP: $\alpha-M T+D O P S)-$
Receiving normal saline $(0.9 \%)$ twice a day at 8 am and $4 \mathrm{pm}$ i.e. at the interval of $8 \mathrm{hrs}$.

5-HTP was administered at 8 am and saline at 4 pm i.e.at the interval of 8 hrs.

III (DOPA control/S:L-DOPA)-

Saline was injected at 8 am and L-DOPA at 4 pm i.e.at the interval of $8 \mathrm{hrs}$.

5-HTP and L-DOPA injections were given $8 \mathrm{hr}$ apart.

5- HTP and PCPA were injected simultaneously at 8 am, and L-DOPA was injected after 8 hrs.

5-HTP was injected at 8 am and $8 \mathrm{hrs}$ later, LDOPA and DDC were injected simultaneously.

5-HTP was injected at 8 am and 8 hrs later, $\alpha$-MT and DOPS were injected simultaneously at $4 \mathrm{pm}$.

and $4 \mathrm{pm}$ so as to establish $8-\mathrm{hr}$ interval/phase relationship between the two injections or set of injections. The dose of 5-HTP and L-DOPA was $5 \mathrm{mg} / 100 \mathrm{~g}$ body weight per day for 13 days. The doses of antagonists were as follows- PCPA (2mg/100 g body wt.) DDC (10 mg/100g body wt.), $\alpha-M T$ (1 mg/100g body wt.) and agonist of noradrenaline DOPS was (10mg/100 g body wt.). During the treatment of 13 days, all the birds were kept in dim continuous light ( 2 lux) and after the treatment period, birds of all the groups were transferred to long day condition (LD 16:8).

The length and width of the cloacal gland was measured in situ with dial calipers weekly; before, during and after the treatment upto the termination of study and cloacal gland volume was calculated in $\mathrm{cm}^{3}$ (Jaiwal and Chaturvedi, 1991; Chaturvedi 
et al., 1993). At the terminations of study i.e. 10 weeks post treatment (at the age of 15 weeks), blood was collected from the alar vein/wing vein into a heparinized tube and centrifuged at $4000 \mathrm{rpm}$ for $20 \mathrm{~min}$ at $4^{\circ} \mathrm{C}$ to separate plasma and stored at $-20^{\circ}$ for hormonal assays to be performed later. Plasma testosterone level was measured using EIA kit (DSI s.r.l., Italy) following manufacturer's protocol. The antiserum used in the assay was $100 \%$ specific for testosterone (cross reactivity/specificity with testosterone was $100 \%$ ); the cross reactivity of the assay was $0.056 \%$ with progesterone, $0.004 \%$ with cortisol, $0.005 \%$ with estradiol, $4.8 \%$ with dihydrotestosterone, $3.6 \%$ with androstenedione, $0.048 \%$ with androsterone, $0.004 \%$ with cortisone, $0.002 \%$ with estriol and $0.007 \%$ with estrone. The analytical sensitivity of the assay was 0.0576 $\mathrm{ng} / \mathrm{ml}$. The intra-assay coefficient of variation $(\mathrm{CV})$ is $5.6 \%$ whereas inter-assay $\mathrm{CV}$ is 7.1\%. Accuracy for this assay was 99\%. Thereafter, birds from each group were weighed, deeply anaesthesized with thiopentone and then dissected to collect tissues so as to process for measuring testicular volume $\left(\mathrm{cm}^{3}\right)$ and for calculating gonadosomatic index-GSI (in gram testes/100 gram body weight). Left testis of each quail after measuring its length and width in situ for calculating testicular volume was fixed in Zamboni's solution and processed for routine histological study and measurement of seminiferous tubule diameter. For histology, twenty-four hours after fixation, the testes were dehydrated in an ascending series of alcohol, treated with xylene and then embedded in paraffin wax. The 6- $\mu$ m thick sections were cut by a Weswox rotary microtome (Western Electric and Scientific Works, Ambala Cantt, India), and stained with hematoxylin-eosin. Histological sections were viewed under a microscope (Axioskop 2 Plus; Carl Zeiss AG, Oberkochen, Germany) and images were captured with a digital camera. The diameter of the seminiferous tubules was determined in 10 sections from each testis by using the occulometer and micrometer. 
All the numerical data (cloacal gland and testicular volume, GSI, seminiferous

tubule diameter and plasma testosterone concentration) were analyzed by one-way

analysis of variance (ANOVA), followed by post hoc Dunnett test for the comparison

of group means. Significance was calculated at the level of $\mathrm{p}<0.05$.

\section{Results}

213 level was observed in saline: saline (S:S) i.e. saline control, HTP: saline i.e. HTP

214 control, saline: DOPA i.e. DOPA control and HTP: $\alpha-\mathrm{MT}+\mathrm{DOPS}$ group quail.

215 However, those of HTP:DOPA, HTP+PCPA:DOPA and HTP:DOPA+DDC remained

216 at significantly low level throughout the period of study compare to control

217 (saline:saline) (Fig. 1). At the termination of study, testicular volume, GSI and plasma

218 testosterone level of HTP:DOPA, HTP+PCPA:DOPA and HTP:DOPA+DDC group

219 quail were significantly lower in comparison to saline control (S:S) but these

220 parameters of other 3 groups were not different from saline control (Fig. 2, 3 and 4).

222 DOPA and HTP:DOPA+DDC 8-hr quail group had smaller seminiferous tubules (Fig.

223 5) with decreased spermatogenesis along with vacuolation in HTP:DOPA and

224 HTP:DOPA+DDC quail and atrophic changes with complete degeneration of 225 spermatogenenic activity was noted in HTP+PCPA:DOPA quail testis unlike full 226 breeding condition in control $(\mathrm{S}: \mathrm{S})$. The interstitial spaces of the seminiferous tubules 227 of these quail testes were reduced and no Leydig cells were evident in triangular spaces. 228 Further, similar to the enlarged seminiferous tubules of saline control quail testis having 229 all the stages of spermatogenesis and bunches of spermatozoa in the lumen, the testis of 
HTP:S, S:DOPA and HTP: $\alpha-M T+D O P S$ also exhibited full breeding condition (Fig. 5

231 and 6).

232

233

234

235

\section{Discussion}

Intraperitoneal injections of 5-HTP and L-DOPA (HTP:DOPA) given $8 \mathrm{hrs}$ apart i.e. 8-hr phase relation of serotonergic and dopaminergic oscillations induced by their precursor injections suppressed gonadal activity significantly compared to saline treated control $(\mathrm{S}: \mathrm{S})$. On the other hand, reproductive parameters of quail receiving saline 8 hrs after 5-HTP (HTP:S) or 8 hrs before DOPA (S:DOPA) serving as HTP control and DOPA control respectively, did not differ from saline control (S:S). This indicates that suppressive effect of 5-HTP and L-DOPA when given $8 \mathrm{hrs}$ apart (8-hr relation) was neither due to 5-HTP nor L-DOPA alone but is actually the outcome of the interval between the administration of serotonergic and dopaminergic precursor drugs inducing 8-hr temporal relationship between the two neural oscillations. In another group of birds, 5-HTP and PCPA was injected simultaneously followed by LDOPA injection at interval $8 \mathrm{hr}$. PCPA blocks the conversion of tryptophan into 5-HTP by inactivating the enzyme tryptophan hydroxylase but it does not affect the conversion of 5-HTP into serotonin. Hence exogenous administration of PCPA blocked the conversion of endogenous tryptophan into 5-HTP, but exogenous 5-HTP was still converted into serotonin. Hence in fact, theoretically 8-hr phase relation of HTP: DOPA should not be different from HTP+PCPA:DOPA and accordingly gonadal response of these two groups are also similar i.e. gonado-suppressive.

In case of HTP:DOPA+DDC quail also, gonadal suppression was observed similar to those quail receiving only two drugs HTP:DOPA 8 hrs apart. The injection of DOPA (dopamine precursor) increases the synthesis of dopamine (DA) and enzyme 
dopamine $\beta$-hydroxylase (DBH) converts DA into noradrenaline (NA). The drug DDC

257 selectively inhibits NA synthesis because it inhibits the enzyme DBH therefore

258 inhibiting the synthesis of NA. Hence simultaneous injection of DOPA and DDC is

259 expected to stimulate dopamine synthesis but inhibits synthesis of NA. Obviously effect

260 of this combination of drugs (HTP:DOPA+DDC) was also similar to that of HTP:

261 DOPA combination indicating that inhibitory effect of 8 -hr temporal relation of

262 precursor drugs was only due to their conversion into serotonin and dopamine and not

263 any further conversion into noradrenaline.

In the HTP: $\alpha-\mathrm{MT}+\mathrm{DOPS}$ group, quail received $\alpha-\mathrm{MT}$ and DOPS simultaneously

8 hrs after 5-HTP administration and the effect was not different from control. The drug

$\alpha$-MT inhibits the conversion of the amino acid tyrosine to DOPA, whereas the drug

DOPS is a specific precursor for NA. Hence, simultaneous injections these two drugs

$(\alpha-M T$ i.e. antagonist of DOPA synthesis and DOPS i.e. agonist of NA) should selectively inhibit synthesis of DOPA (and hence also the dopamine synthesis) and increase NA synthesis respectively. Because in this treatment of HTP: $\alpha-M T+D O P S$, there is no exogenous DOPA (precursor of dopamine) and internal source of DOPA is also blocked by $\alpha-\mathrm{MT}$, the DA synthesis is expected to be blocked completely but NA is still available synthesized from its selective precursor DOPS. Although natural

274 precursor of adrenaline/NA i.e. dopamine is not available (as endogenous dopamine 275 synthesis have been blocked by $\alpha$-MT, and exogenous DOPA is not available), but NA 276 will be still available because of injecting its selective precursor DOPS. Thus this combination of drugs is equivalent to 8 -hr relationship between 5 -HTP/serotonin and

278 NA (noradrenaline) and not the 5-HTP/ serotonin and L-DOPA /dopamine. But, in

279 terms of gonadal response, effect is similar to HTP control which in turn is similar to 280 saline control. 

(dopamine and noradrenaline) suggest that reproductive effect of 5-HTP and L- DOPA

287 given 8 hrs apart apparently result from their conversion into serotonin and dopamine and not due to other monoamine/catecholamine. Selective potentiation of dopaminergic activity by DOPA and DDC injections did suppress reproductive development whereas specific inhibition of dopaminergic activity by $\alpha$-MT and stimulation of noradrenaline by DOPS injection did not. Therefore, HTP/ /serotonergic or L-DOPA/dopaminergic activity independently is unable to induce any change in gonadal activity which is actually the effect/function of interval between the administration of neurotransmitter precursor drugs 5-HTP and L- DOPA. Moreover, it is not the dose of neurotransmitter precursor drugs (5-HTP and L-DOPA) that cause the effect, instead it is the specific time interval between the drug administrations, otherwise the effects could have been similar in all cases and not the one observed in all the reports consistently. Invariably in many species, 8-hr phase relation is gonado-suppressive, 12-hr is gonado-stimulatory and other relations are ineffective (Chaturvedi and Bhatt, 1990; Chaturvedi and Yadav, 2013).

Based on these findings it is restated that effects observed in the experiments of present study, are due to specific phase relation of serotonergic and dopaminergic oscillations induced by systemic injections of 5-HTP and L- DOPA given at different interval. Present findings also suggest that the observed effect are due to conversion of DOPA into dopamine and not into the next biosynthetic product (NA). 


\section{Acknowledgements}

Junior research fellowship to Suneeta Yadav from University Grants Commission (BSR-RFSMS), New Delhi and Research projects to CMC from Department of science and Technology, New Delhi, India (SR/50/AS-14/2012) is gratefully acknowledged.

\section{References}

1. Bhatt, R. and Chaturvedi, C.M. (1992a). Specific phase relation (8-hr) of Neurotransmitter precursor drugs alters body weight, plasma thyroxine, testes and cloacal gland responses of Japanese quail. PAVO. 30 (1\&2), 9-16.

2. Bhatt, R. and Chaturvedi, C.M. (1992b). The effects of combined serotonin and dopamine precursor application during testicular photosensitivity vs photofractoriness of the Red headed bunting, Emberiza bruniceps. J. Interdiscipl. Cycle Res. 23 (2), 120-127.

3. Bhatt, R. and Chaturvedi, C.M. (1998). Phase relation of neurotransmitter affecting drugs (5-HTP and L-DOPA) alters photo-sexual responses (scotosensitive and scotorefractory) in Japanese quail. J. Environ. Biol. 19, 285-293.

4. Bianchine, J.R. (1980). Drugs for Parkinson's diseases. In: Goodman, L.S., Gilman, A.G, (eds.), The Pharmacological Basis of Therapeutics, $6^{\text {th }}$ edn. Macmillan, New York, pp. 475-493.

5. Chaturvedi, C.M. and Bhatt, R. (1990). The effects of different temporal relationships of 5-hydroxytyptophan (5-HTP) and L-dihydroxyphenylalanine (LDOPA) on reproductive and metabolic responses of migratory Red Headed Bunting (Emberiza bruniceps). J. Interdiscipl. Cycle Res. 21, 129-139.

6. Chaturvedi, C.M. and Jaiwal, R. (1990). Temporal synergism of neurotransmitter affecting drugs \& seasonal reproductive responses of Indian Palm squirrel, Funambulus pennanti. J. Neural Transm. 81, 31-40. 
7. Chaturvedi, C.M. and Prasad, S.K. (1991). Timed daily injections of Neurotransmitter precursor alter the gonad and body weights of Spotted munia, Lonchura punctulata, maintained under short daily photoperiods. J. Exp. Zool. 260, 194-201.

8. Chaturvedi, C.M. and Singh, A.B. (1992). Suppression of annual testicular development in Indian Palm squirrel, Funambulus pennanti by 8-hr temporal relationship of serotonin and dopamine precursor drugs. J. Neural Transm. 88, 5360.

9. Chaturvedi, C.M. and Yadav, S. (2013). Influence of temporal relationships between serotonergic and dopaminergic precursors on the regulation of gonadal development in birds. Gen. Comp. Endocrinol. 190, 203-213.

10. Chaturvedi, C.M., Meier, A.H. and Bhatt, R. (1991). Effect of 12-hr temporal relation of serotonin and dopamine precursor drugs (5-HTP and L-DOPA) on photo-sexual responses of immature Japanese quail. Ind. J. Exp. Bio. 29, 342-345.

11. Chaturvedi, C.M., Bhatt, R. and Phillips, D. (1993). Photoperiodism in Japanese quail with special reference to relative refractoriness. Ind. J. Exp. Biol. 31, 417421.

12. Chaturvedi, C.M., Bhatt, R. and Prasad, S.K. (1994). Effect of timed administration of neurotransmitter drugs on testicular activity, body weight and plumage pigmentation in the Lal munia, Estrilda amandava. Ind. J. Exp. Biol. 32, 238-242.

13. Chaturvedi, C.M., Das, U.S. and Thapliyal, J.P. (1997). Comparative aspects in the reproductive endocrinology of the two sexes of weaver bird, Ploceus philippinus. In: Kawashima, S., Kikuyama, S. (Eds.), Advances in Comparative Endocrinology, Proceedings XIII Intern. Cong. Comp. Endocrinol., Japan, pp 451-455. 
14. Chaturvedi, C.M., Tiwari, A.C. and Kumar, P. (2006). Effect of temporal synergism of neural oscillations on photorefractoriness in Japanese quail (Coturnix coturnix japonica). J. Exp. Zool. 305A, 3-12.

15. Crevelling, C.R., Daily, J., Tikuyama, T. and Wittkip, B. (1968). The combined use of $\alpha$-methy-tyrosine and threodihydroxyphenylserine: Selective reduction of dopamine levels in the central nervous system. Biochem. Pharmacol. 17, 65-70.

16. Forsling, M.L. (2000). Diurnal rhythms in neurohypophysial function. Exp Physiol. $85,179 \mathrm{~S}-186 \mathrm{~S}$

17. Héry, M., Dusticier, G., Faudon, M., Barrit, M.C. and Hey, F. (1981). Kinetic study of serotonin metabolism in the suprachiasmatic nucleus of the rat: neuroendocrine incidence (author's transl). J Physiol (Paris). 77 (2-3), 497-500.

18. Jaiwal, R. and Chaturvedi, C.M. (1991). Elimination of testicular regression by $12-$ hr temporal relationship of serotonergic and dopaminergic activity in Indian Palm squirrel, Funambulus pennanti. J. Neural Transm. 84, 45-52.

19. Khan, I.A. and Joy, K.P. (1990). Effects of season, pinealectomy, and blinding alone and in combination, on hypothalamic monoaminergic activity in the teleost Channa punctatus (Bloch). J Pineal Res. 8 (3), 277-287.

20. Koe, B.K. and Weissman, A. (1966). p-chlorophenylalanine: a specific depletory of brain serotonin. J. Pharmacol. Exp. Ther. 154 (3), 499-516.

21. Kumar, P. and Chaturvedi, C.M. (2008). Correlation of nitric oxide (NO) activity and gonadal function in Japanese quail, Coturnix coturnix japonica following temporal phase relation of serotonergic and dopaminergic oscillations. Anim. Reprod. Sci. 106, 48-64. 
22. Le Bras, Y.M. (1984). Circadian variations of catecholamine levels in brain, heart, and plasma in the eel, Anguilla Anguilla, at three different times of year. Gen Comp Endocrinol. 55(3), 472-479.

23. Lynch, H.J. (1971). Diurnal oscillations in pineal melatonin content. Life Sci. 10 (14), 791-795.

24. Manshardt, J. and Wurtman, R.J. (1968). Daily rhythm in the noradrenaline (norepinephrine) content of rat hypothalamus. Nature. 217 (5128). 574-575.

25. Owasoya, J.O. and Walker, C.A. (1979). Whitworth UG. Diurnal variation in the dopamine level of rat brain areas: effect of sodium Phenobarbital. Life Sci. 25 (2),

$$
119-122 \text {. }
$$

26. Owasoyo, J.O. and Walker, C.A. (1980). The effect of sodium phenobarbital on the circadian levels of norepinephrine and serotonin in rat brain areas. Biol Rhy Res. 11

$$
\text { (2), 95-101. }
$$

27. Phillips, D. and Chaturvedi, C.M. (1992b). Photo-sexual responses of young Japanese quail following circadian administration of neurotransmitters. In: Pati, A. K. (Ed.), Chronobiology, RSU, Raipur, India, pp. 73-79.

28. Phillips, D. and Chaturvedi, C.M. (1995). Functional maturation of neuroendocrine gonadal axis is altered by specific phase relations of circadian neurotransmitter activity in Japanese quail. Biomedical and Environment Sciences. 8, 367-377.

29. Philo, R., Rudeen, P.K. and Reiter, R.J. (1977). A comparison of the circadian rhythms and concentrations of serotonin and norepinephrine in the telencephalon of four rodent species. Comp Biochem Physiol C. 57 (2), 127-130.

30. Prasad, S.K. and Chaturvedi, C.M. (1992a). Circadian phase relation of neurotransmitter affecting drugs (serotonergic and dopaminergic) alters the gonadal 
activity during quiescent phase of reproductive cycle in Spotted Munia, Lonchura punctulata. Int. J. Animal. Sci. 7, 127-129.

31. Prasad, S.K. and Chaturvedi, C.M. (1992b). Negation of Arhythmic cyclicity by circadian phase relation of serotonergic and dopaminergic activity in a subtropical finch, Spotted Munia. In: Saxena, A.K., Ramamurty, R., Reddy Sreerama, G., Saxena, V.L. (Eds.), Recent Advances in Life Sciences, Indian Society of Life Sciences, Manu Publications, Kanpur, U.P. India, pp 90-99.

32. Prasad, S.K. and Chaturvedi, C.M. (1992c). Effects of LH and temporal synergism of neurotransmitter affecting drugs (5-HTP and L-DOPA) on the gonad and body weight responses of spotted Munia Lonchura punctulata. J. Rep. Biol. Comp. Endocrinol. 4 (1), 1-8.

33. Prasad, S.K. and Chaturvedi, C.M. (2003). Regulation of seasonal reproduction by neurotransmitter affecting drugs in spotted munia, Lonchura punctulata. Pakistan J. Zool. 35 (3), 181-189.

34. Quay, W.B. (1968). Differences in circadian rhythms in 5-hydroxytryptamine according to brain region. Am J Physiol. 215(6), 1444-1453.

35. Reis, D.J. and Wurtman, R.J. (1968). Diurnal changes in brain noradrenalin. Life Sci. 7 (1), 91-98.

36. Reis, D.J., Weinbren, M. and Corvelli, A. (1969). A circadian rhythm of norepinephrine regionally in cat brain: its relationship to environmental lighting and to regional diurnal variations in brain serotonin. J Pharmacol Exp Ther. 164 (1), 135-145.

37. Saito, Y. (1971). The circadian rhythm of brain acetylcholine levels and motor activity in the rat. Life Sci. 10 (13), 735-744, 
426 38. Sethi, S. and Chaturvedi, C.M. (2009). Temporal synergism of neurotransmitters

427 (Serotonin and Dopamine) affects testicular development in mice (Mus musculus).

$428 \quad$ Zoology. $112(6), 461-470$.

429 39. Sethi, S., Tsutsui, K. and Chaturvedi, C.M. (2010). Temporal phase relation of 430 circadian neural oscillations alters RFamide-related peptide -3 and testicular 431 function in the mouse. Neuroendocrinol. 91, 189-199.

432 40. Tiwari, A.C. and Chaturvedi, C.M. (2003). Effect of temporal synergism of neural 433 oscillators on gonadal development, egg production and hatchability of Japanese 434 quail (Coturnix coturnix). Indian J. Poul. Sci. 38 (2), 83-88.

435 41. Tiwari, A.C., Kumar, P., Singh, S., Sharma, D. and Chaturvedi, C.M. (2006). 436 Reproductive phase dependent circadian variation in hypothalamic concentration of 437 serotonin, dopamine and peripheral thyroxine levels in Japanese quail following 5$438 \quad$ HTP and L- DOPA administration at specific time intervals. Biol. Rhythm Res. 37 (1), 73-86.

42. Wilson, J.M. and Meier, A.H. (1987). Seasonal changes in the circadian rhythms of serotonin and dopamine concentration in right and left supra chiasmatic nuclei (SCN) of Syrian hamster. Chronobiol. 14, 255.

443 43. Wilson, J.M. and Meier, A.H. (1988). Seasonal changes in the phase relations of 444 circadian neurotransmitter rhythms in the anterior hypothalamus (Abstract). IEEE Transaction. 4, 1820-1821.

446 44. Wilson, J.M. and Meier, A.H. (1989). Resetting the annual cycle with timed daily 447 injections of 5-hydroxytryptophan and L- dihydroxyphenylalanine in Syrian 448 hamsters. Chronobiology International. 6, 113-132. 
449 45. Yadav, S. and Chaturvedi, C.M. (2014). Internal coincidence of serotonergic and

450 dopaminergic oscillations modulates photo-sexual responses of Japanese quail,

451 Coturnix coturnix japonica. Ind J Exp Biol. 52 (5), 489-495

452 46. Yadav, S. and Chaturvedi, C.M. (2015). Interaction of specific temporal phase

453 relations of circadian neural oscillations and long term photoperiodic responses in

$454 \quad$ Japanese quail, Coturnix coturnix japonica. Gen Com Endocrinol. 54-6, 217-218. 


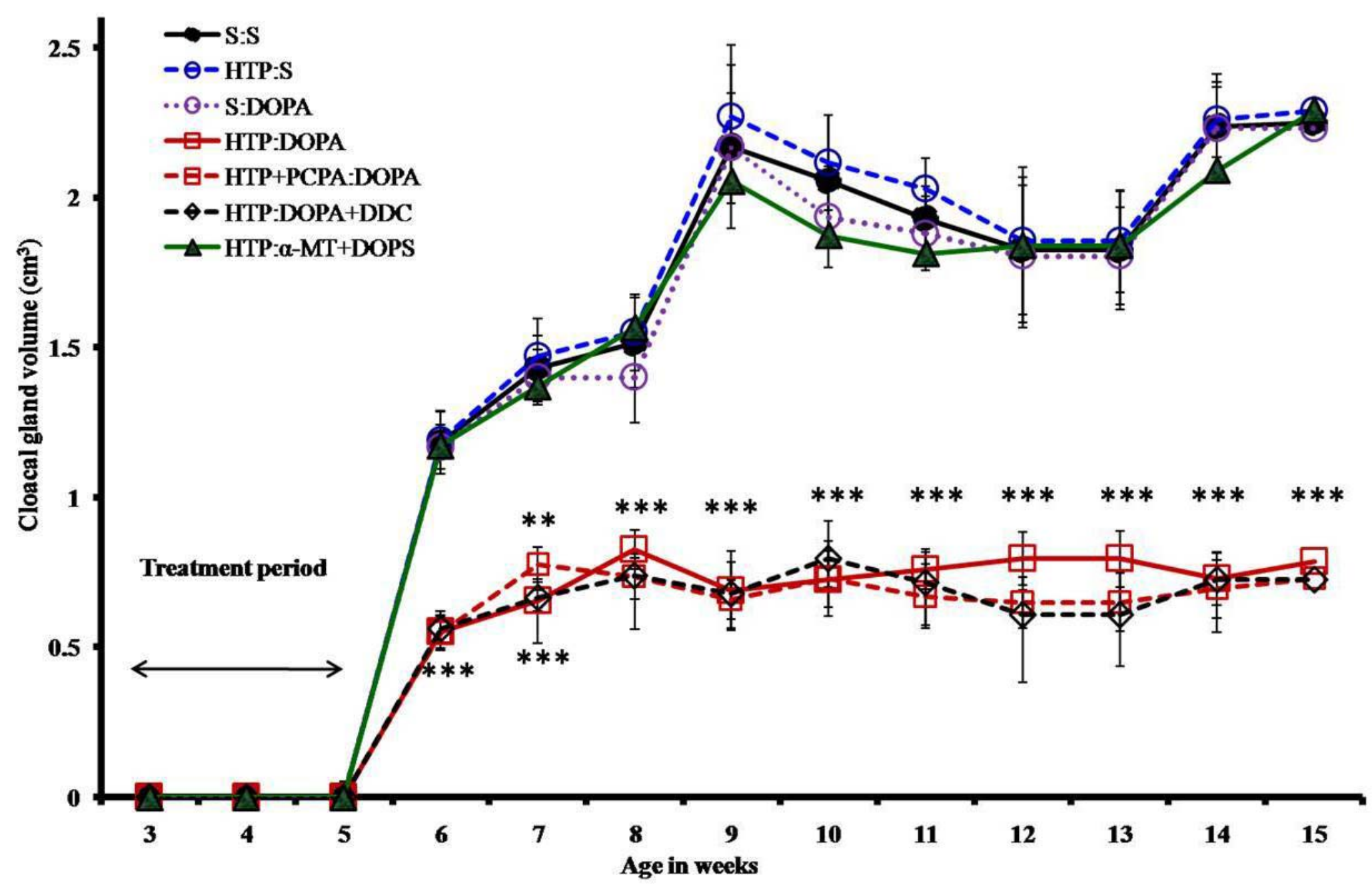


Fig. 1. Cloacal gland responses of Japanese quail receiving 5-HTP and L-DOPA in combination with saline and agonist and/or antagonist of serotonin and catecholamines 8 hrs apart.

Data is presented as means \pm SEM. $* * p<0.01$, ***p $<0.001$, Significance of difference from the control group.

Group details:

Saline control/S:S

- Received normal saline (0.9\%) twice a day at $8 \mathrm{am}$ and $4 \mathrm{pm}$ i.e. at the interval of 8 hrs.

HTP control/HTP:S

- 5-HTP was administered at 8 am and saline at 4 pm i.e. at the interval of $8 \mathrm{hrs}$.

DOPA control/S:DOPA

- Saline was injected at 8 am and L-DOPA at 4 pm i.e. at the interval of $8 \mathrm{hrs}$.

HTP:DOPA -5-HTP and L-DOPA injections were given $8 \mathrm{hr}$ apart.

HTP+PCPA:DOPA -received 5- HTP and PCPA simultaneously at 8 am, and L-DOPA was injected after 8 hrs.

HTP:DOPA+DDC

-5-HTP was injected at 8 am and 8 hrs later, L-DOPA and DDC were injected simultaneously.

HTP: $\alpha-M T+D O P S$

-5-HTP was injected at 8 am and 8 hrs later, $\alpha$-MT and DOPS were injected simultaneously at $4 \mathrm{pm}$. 


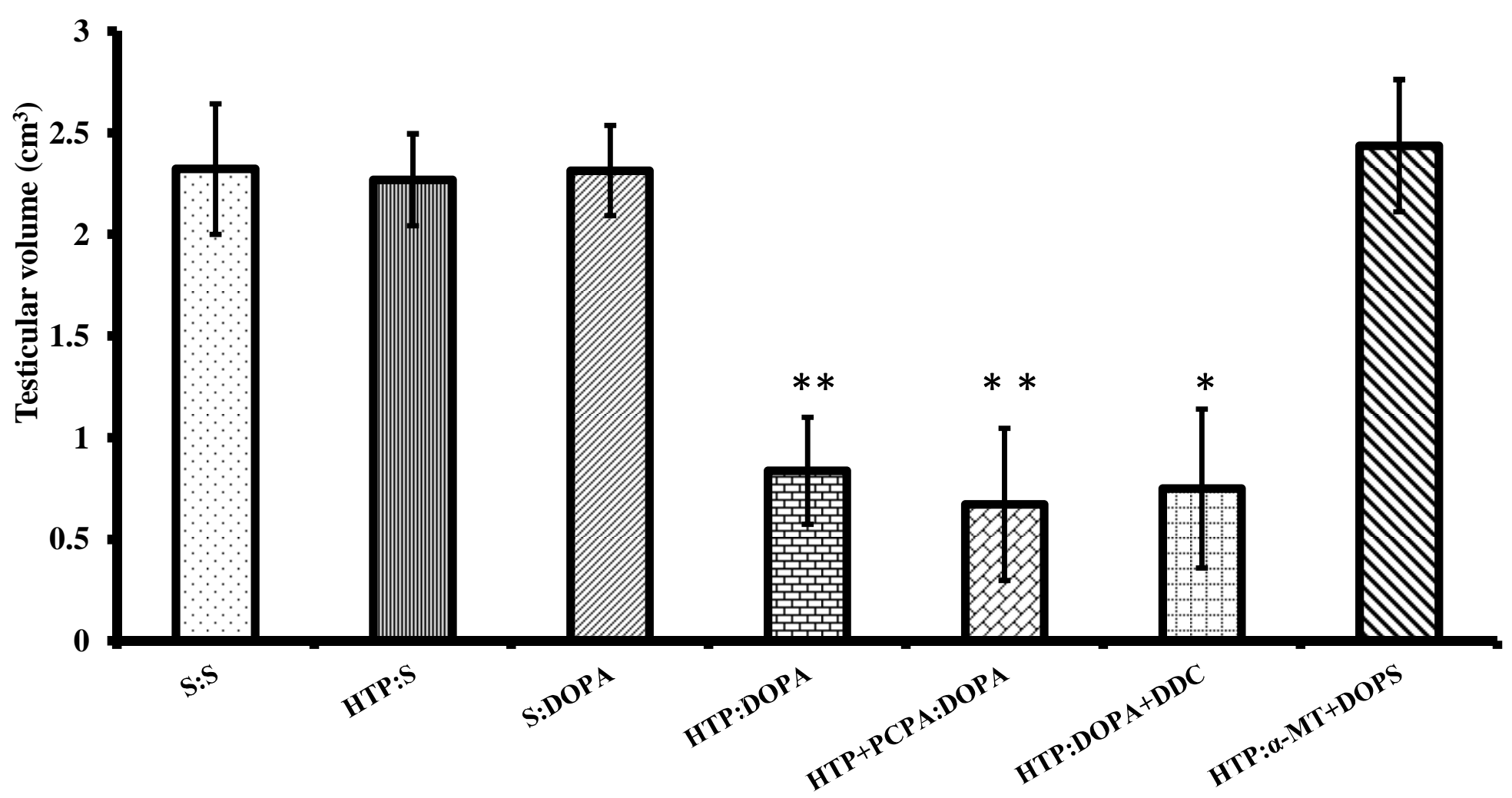

Fig. 2. Testicular volume of Japanese quail receiving 5-HTP and L-DOPA in combination with saline and agonist and/or antagonist of serotonin and catecholamine 8 hrs apart. For group details, see Fig. 1. Data is presented as mean \pm SEM. ${ }^{*} \mathrm{p}<0.05$, ** $<<0.01$; Significance of difference from the control (S:S) group. 


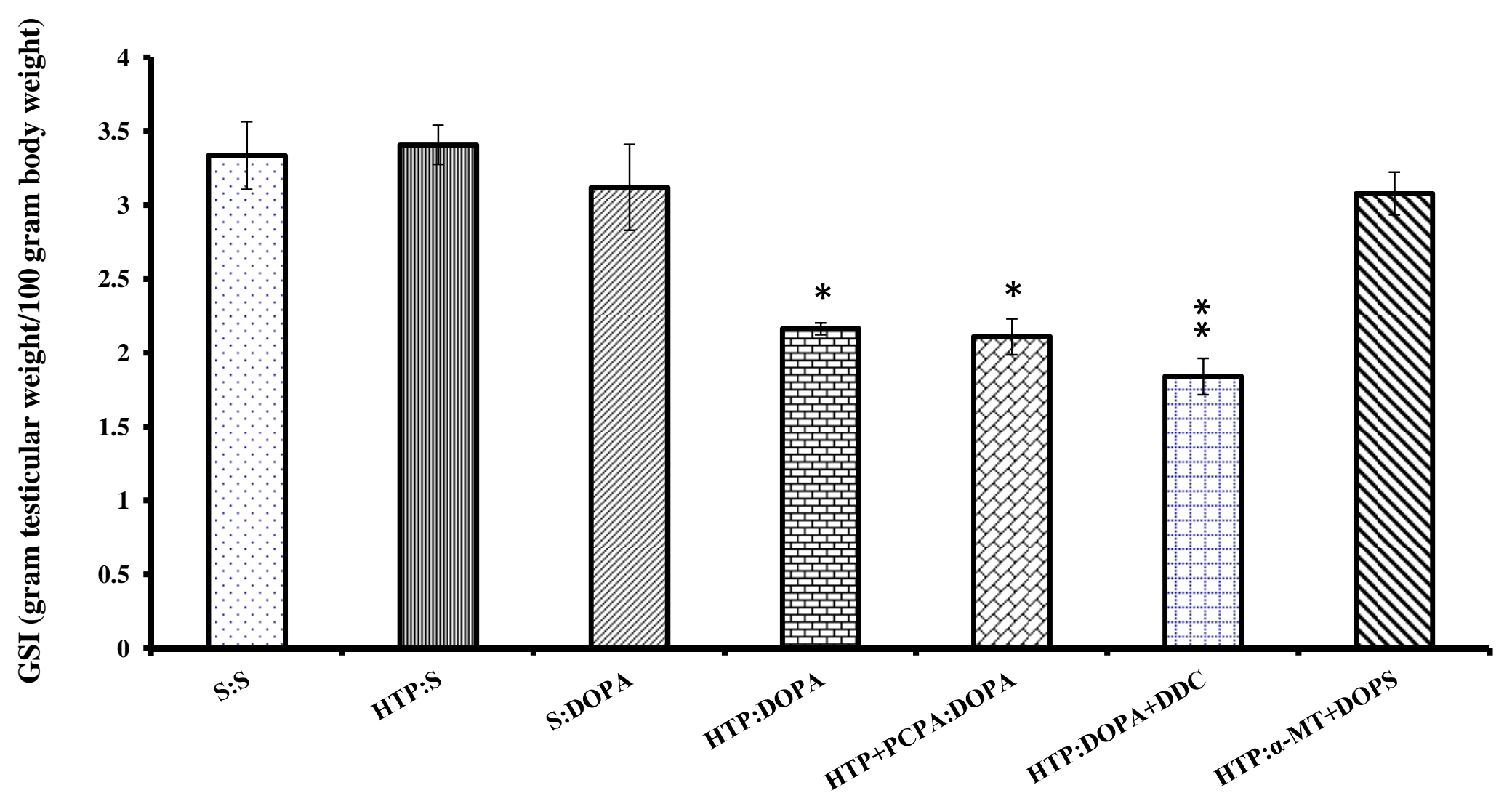

Fig. 3. GSI (Gonadosomatic index) of Japanese quail receiving 5-HTP and L-DOPA in combination with saline and agonist and/or antagonist of serotonin and catecholamine 8 hrs apart. For group details, see Fig. 1.

Data is presented as mean \pm SEM. ${ }^{*} p<0.05,{ }^{*} p<0.01$; significance of difference from the control group 


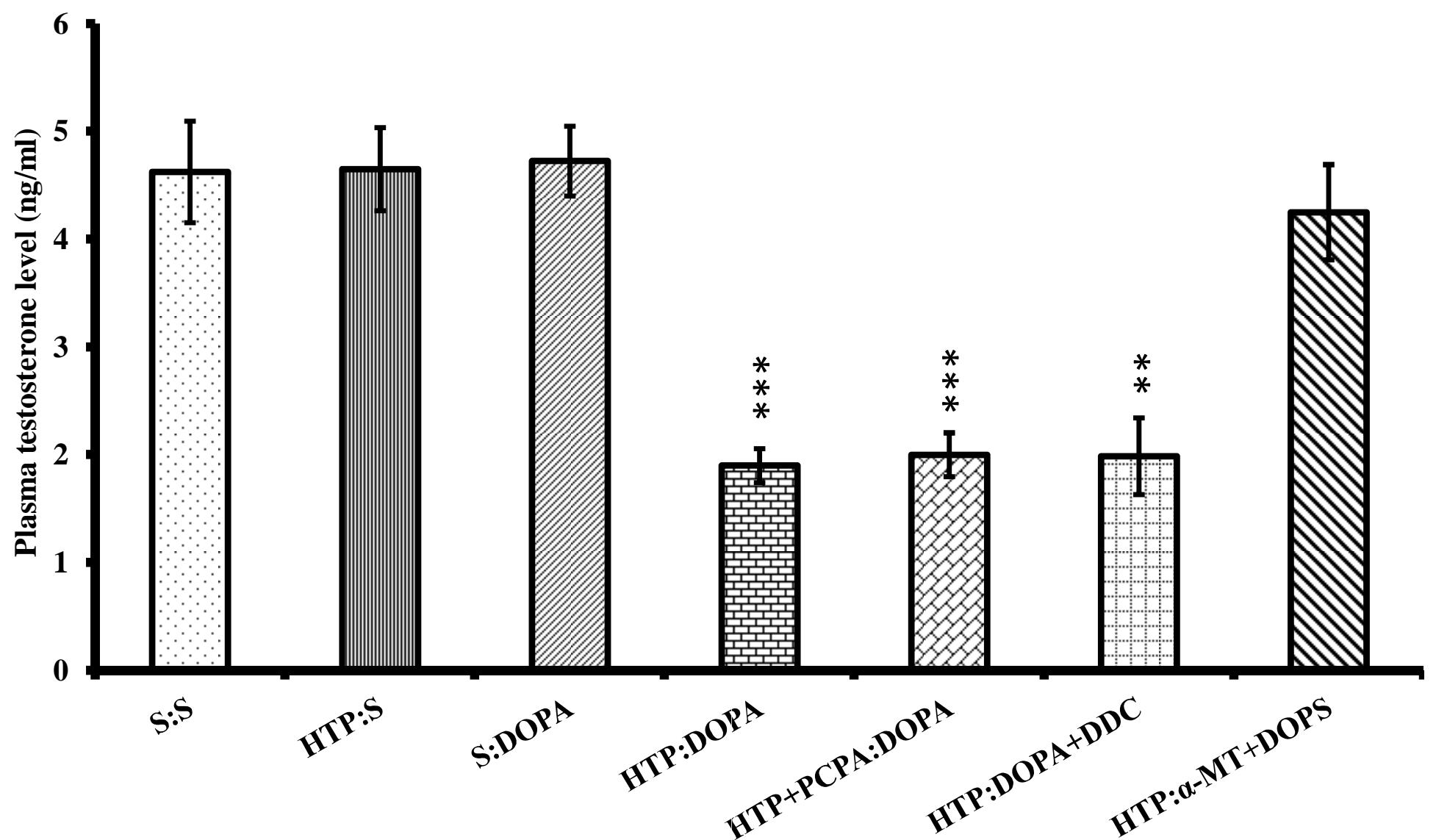

Fig. 4. Plasma testosterone level of Japanese quail receiving 5-HTP and L-DOPA in combination with saline and agonist and/or antagonist of serotonin and catecholamine $8 \mathrm{hrs}$ apart. ${ }^{* *} \mathrm{p}<0.01, * * * \mathrm{p}<0.001$; Significance of difference from the control group. 


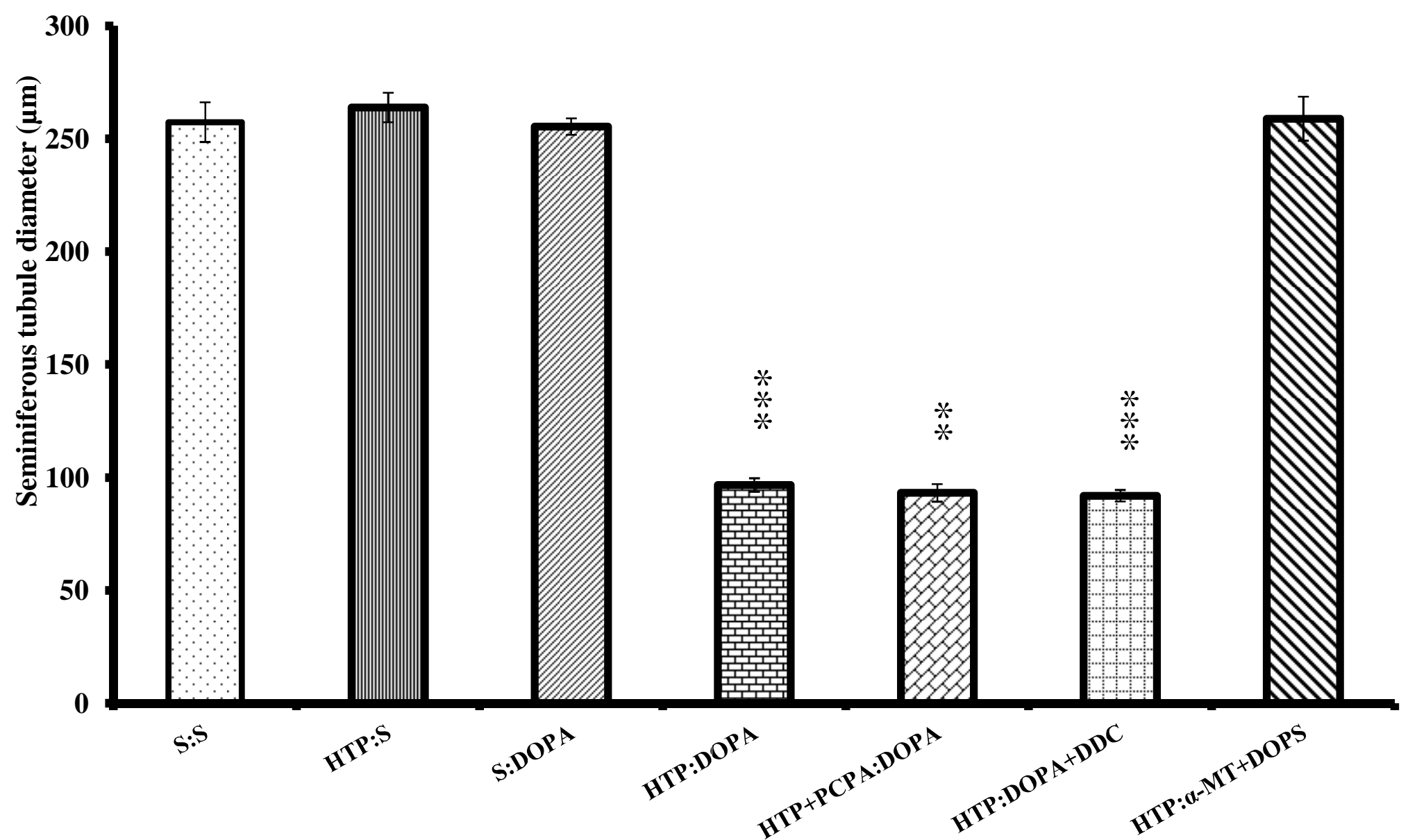

Fig. 5. Seminiferous tubule diameter of Japanese quail receiving 5-HTP and L-DOPA in combination with saline and agonist and/or antagonist of serotonin and catecholamine 8 hrs apart. **p $<0.01$, ***p $<0.001$; Significance of difference from the control group. 


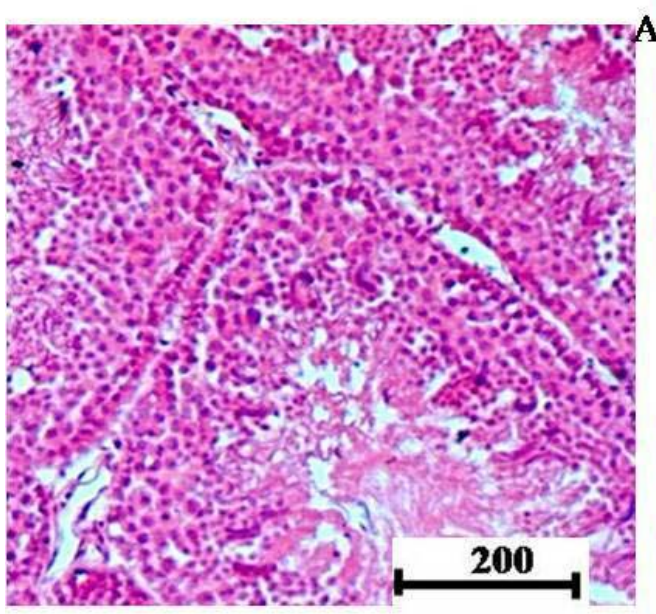

Control (S:S)

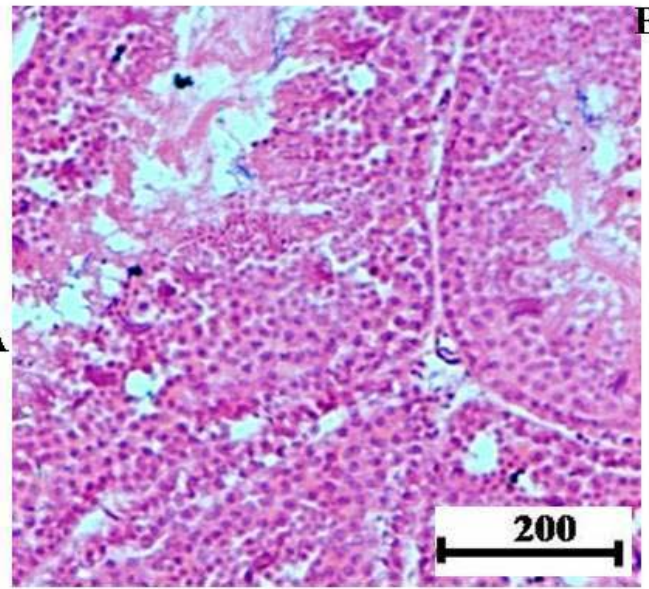

HTP:S

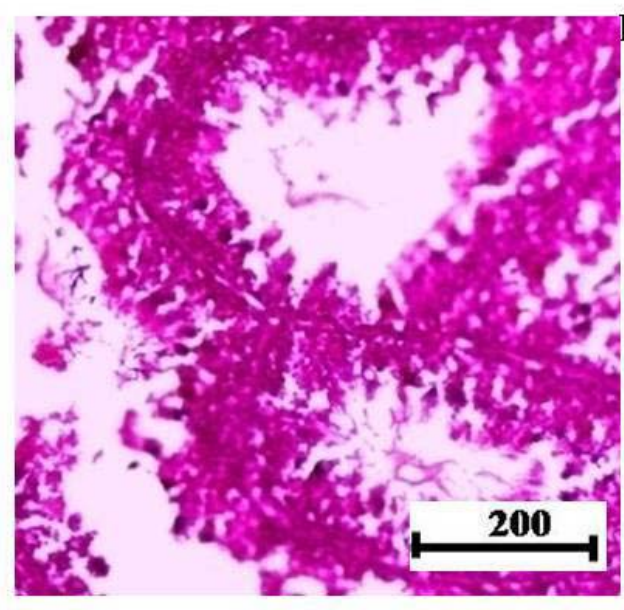

HTP+PCPA:DOPA

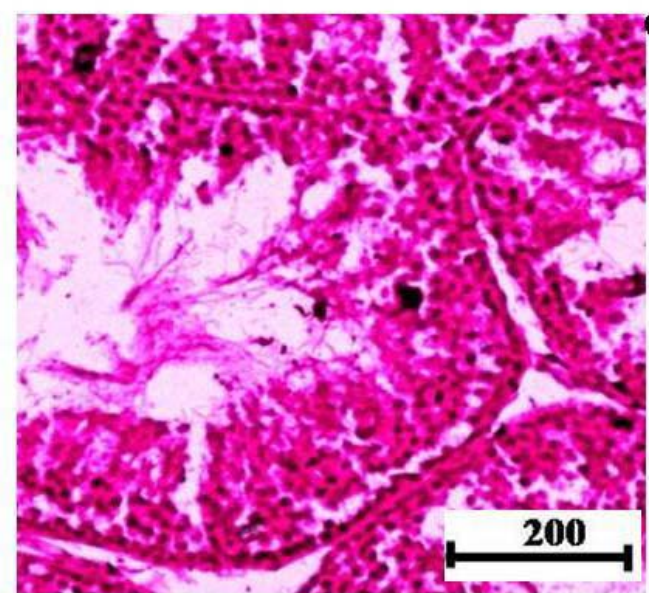

S:DOPA

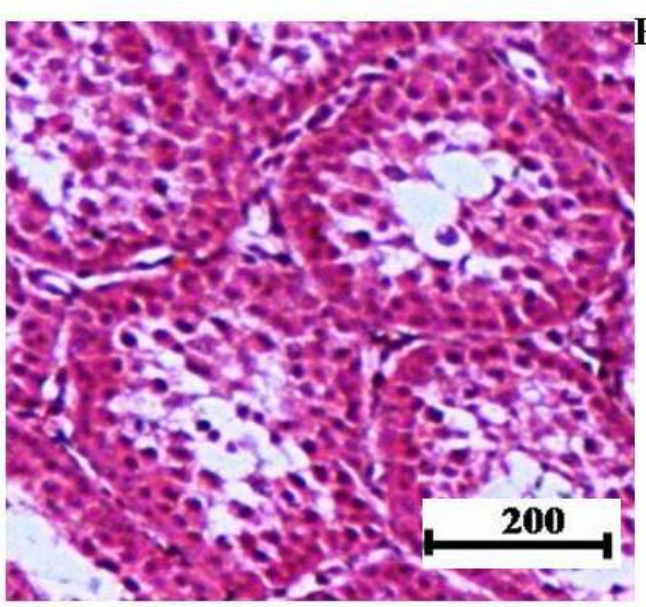

HTP:DOPA+DDC

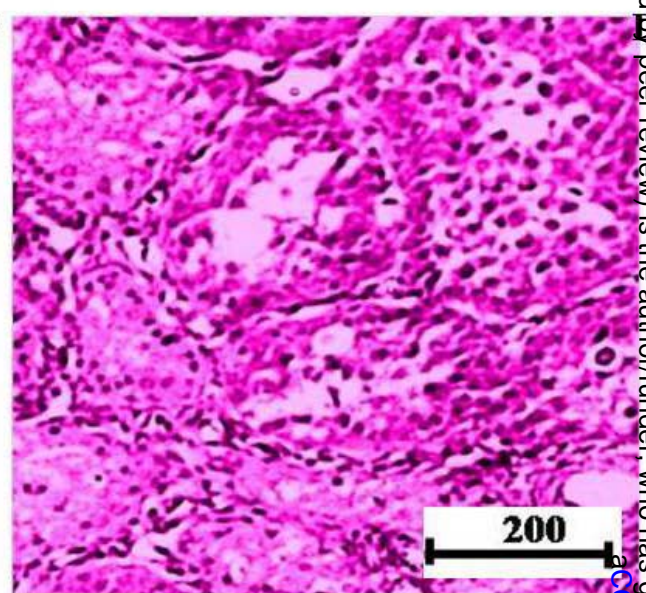

HTP:DOPA

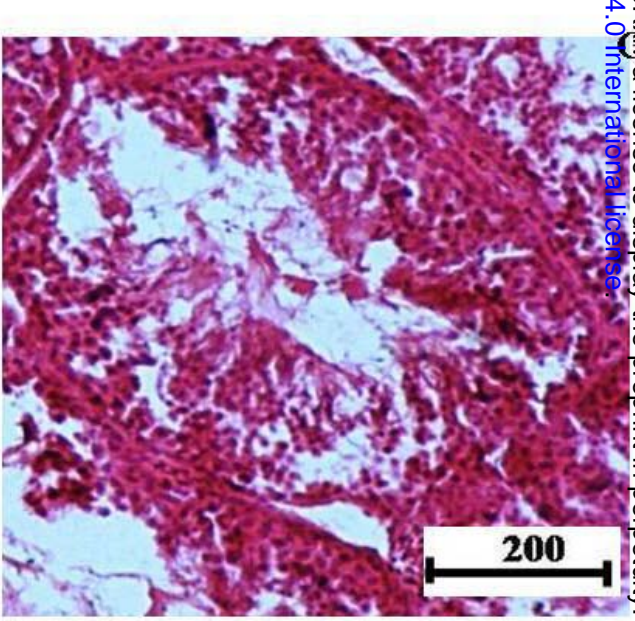

HTP:a-MT+DOPS 
Fig. 6. T.S. of testis of Japanese quail receiving 5-HTP and L-DOPA in combination with saline and agonist and/or antagonist of serotonin and catecholamines 8 hrs apart.

A. Control (S:S)- Note full breeding condition of testis having enlarged seminiferous tubules with all the stages of spermatogenesis and spermatozoa in the lumen.

B. HTP control (HTP:S)- showing full breeding condition as in S:S quail testis.

C. DOPA control (S:DOPA)- showing full breeding condition as in S:S quail testis.

D. HTP:DOPA- Note non breeding condition with smaller seminiferous tubules containing only inactive spermatogonial cells and some vacuolation and debrises in the lumen of few tubules

E. HTP+PCPA:DOPA- Note smaller seminiferous tubules with suppression of stages of spermatogenic activity and emply lumen or some debrises in lumen .

F. HTTP:DOPA+DDC- Note non-breeding and spermatogenetically inactive condition having smaller seminiferous tubules containing inactive spermatogonial cells and vacuolation.

G. HTP: $\alpha$-MT+DOPS group receiving 5-HTP and $\alpha$-MT +DOPS at the interval of $8 \mathrm{hr}$. This section shows normal breeding condition like control. 